\title{
Clinico-Dermoscopic Aspect and Surgical Procedure of Glomus Tumor
}

\author{
Safae Zinoune ${ }^{\star}$, Hanane Baybay ${ }^{1}$, Sara Dahouki ${ }^{1}$, Sara Elloudi' ${ }^{1}$ Zakia Douhi ${ }^{1}$, Mouna Rimani ${ }^{2}$ and Fatima-Zahra \\ Mernissi $^{1}$ \\ IDepartment of Dermatology, Hassan II University Hospital, Fez, Morocco \\ ${ }^{2}$ Hassan Center of Anatomopathology, Rabat, Morocco
}

\section{Article Info}

\section{*Corresponding author:}

Safae Zinoune

Department of Dermatology

Hassan II University Hospital

Fez, Morocco

E-mail: dr.zinounesafae@gmail.com

Received: April 3, 2019

Accepted: April 19, 2019

Published: April 29, 2019

Citation: Zinoune S, Baybay H, Dahouki S, et al. Clinico-Dermoscopic Aspect and Surgical Procedure of Glomus Tumor. Madridge J Surg. 2019; 2(1): 62-66

doi: 10.18689/mjs-1000115

Copyright: @ 2019 The Author(s). This work is licensed under a Creative Commons Attribution 4.0 International License, which permits unrestricted use, distribution, and reproduction in any medium, provided the original work is properly cited.

Published by Madridge Publishers

\begin{abstract}
Glomus tumors are a benign and vascular hamartoma that originates from the neuromyoarterial cells of the normal glomus apparatus in the reticular dermis. They are relatively rare and have a particular tropism for the nail system. Pain is the master symptom but the diagnosis confirmation is based on histology. The use of nail plate and intraoperative dermoscopy facilitates the diagnosis and helps in the localization and demarcation of the tumor. Complete surgical excision is the curative treatment of choice for these tumors. The transungual method is safe and effective, allowing better visualization, easy exploration, and minimal long-term complications. We report a case of glomus tumor developed in the matrix dermis, and treated transungually with partial proximal nail avulsion. Through this case, we emphasize the pre and intraoperative dermoscopic features, and we detail operative procedure.
\end{abstract}

Keywords: Glomus Tumor; Nails; Dermoscopy; Surgical procedure; Histology.

\section{Introduction}

Glomus tumors (GT) are considered to be a hamartoma developing from the neuromyoarterial glomus body, which is a highly specialized arteriovenous anastomosis responsible for thermoregulation [1]. GT are extremely rare benign tumors, accounting for $1 \%$ to $4.5 \%$ of all hand tumors [2]. They may affect any area of the body, but up to $75 \%$ occur in the hand and approximately $65 \%$ of these are in the fingertips, particularly in the subungual space [3]. In general, GT are most commonly seen in female patients in the subungual region in the hands, while men often experience these tumors in other body parts [4]. Diagnosis is mainly on clinical grounds although Magnetic resonance imaging (MRI) has been reported to be useful in cases with multiple lesions and in unusual locations [5]. The complete surgical excision is the only effective treatment modality, however, the risk of post-operative nail deformity and recurrences are the major concerns in this regard [6]. We present our experience with the management of subungual glomus tumors using the transungual excision approach with partial proximal nail avulsion.

\section{Case Report}

A 48-year-old female, physiotherapist, consulted for spontaneous lancinating pain in front of the right thumbnail that was getting more painful when her digit was exposed to cold. Furthermore, the tip was excessively sensitive to touch, and her pain increased at night. On examination, there was an aspect of discrete longitudinal erythronychia (Figure 1A), and elective pain at distal matrix pressure. Onychoscopy of the nail plate 
showed longitudinal erythronychia with disappearance of the lunula opposite the red band and discrete linear vascular structures (Figure 1B). The diagnosis of glomus tumor was therefore evoked. A Magnetic resonance imaging of the right thumb was requested objecting the presence of a small, limited, hyperintense mass in T2 of $45 \times 25 \mathrm{~mm}$ on the proximal matrix (Figure2). Surgical excision was recommended.

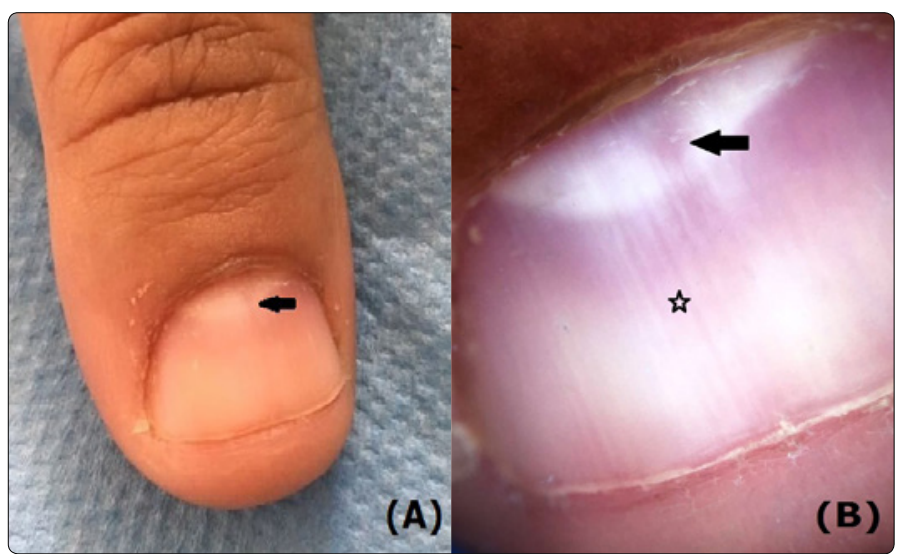

Figure 1. (A) Discrete longitudinal erythronychia on the right thumb nail (arrow) (B) Polarized light dermoscopy of the nail plate show longitudinal erythronychia, partial disappearance of the lunula

(arrow) and discrete linear vascular structures (star).

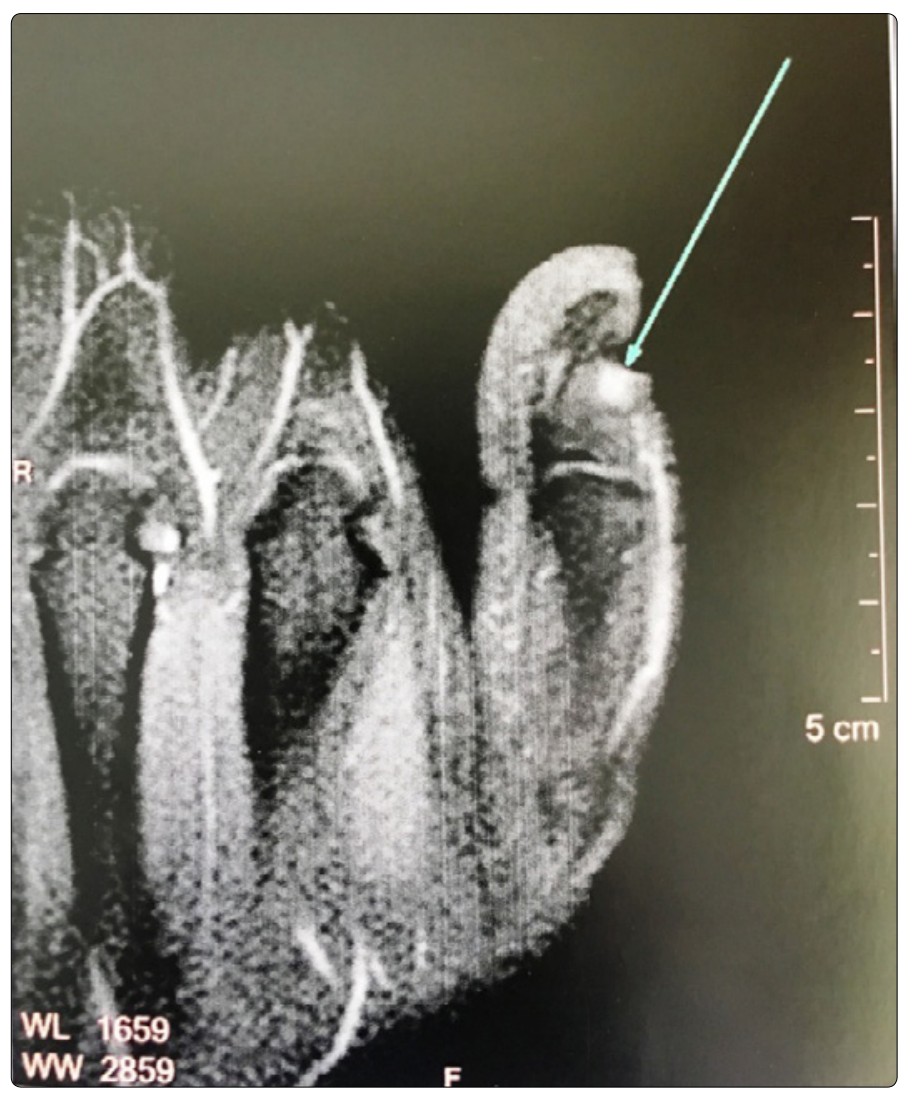

Figure 2. MRI showing a tumor at the subungual aspect of distal phalanx which is hyperintense on $\mathrm{T} 2$ weighted images.

The surgical site was prepared antiseptically. The digit to be operated was anesthetized with proximal nerve block using the $2 \%$ lidocaine hydrochloride solution. Digital exsanguination and tourniquet for relatively bloodless operating field were done, then the proximal nail fold (PNF) was retracted by lateral release incisions and stay sutures (Figure 3). The realization of a partial proximal nail avulsion had made it possible to expose the matrix tumor which appeared under the matrix epithelium in the form of a small rosy lesion (Figure 4). Intraoperative noncontact dermoscopy revealed a purple well-limited tumor (Figure 5A). The epithelium of the distal matrix was incised transversely with detachment under the epidermis and then around the tumor (Figure 5B). The nodule was very meticulously dissected from the surrounding tissue with blunt curved scissors, and then it was removed (Figure 6A). Exvivo dermoscopy of the removed nodule revealed a pinkish homogeneous nodule with marked telangiectasias (Figure 6B). The residual cavity is sutured with the rapid absorbable thread $6 / 0$ (Figure 7A). The nail plate was repositioned to protect the surgical site and lateral incisions were sutured with non-absorbable thread 3-0, which was removed after 15 days (Figure 7B). The histopathology of the tumor confirmed the diagnosis of GT (Figure 8). There is no pain nail deformation or recurrence at one year follow-up (Figure 9).

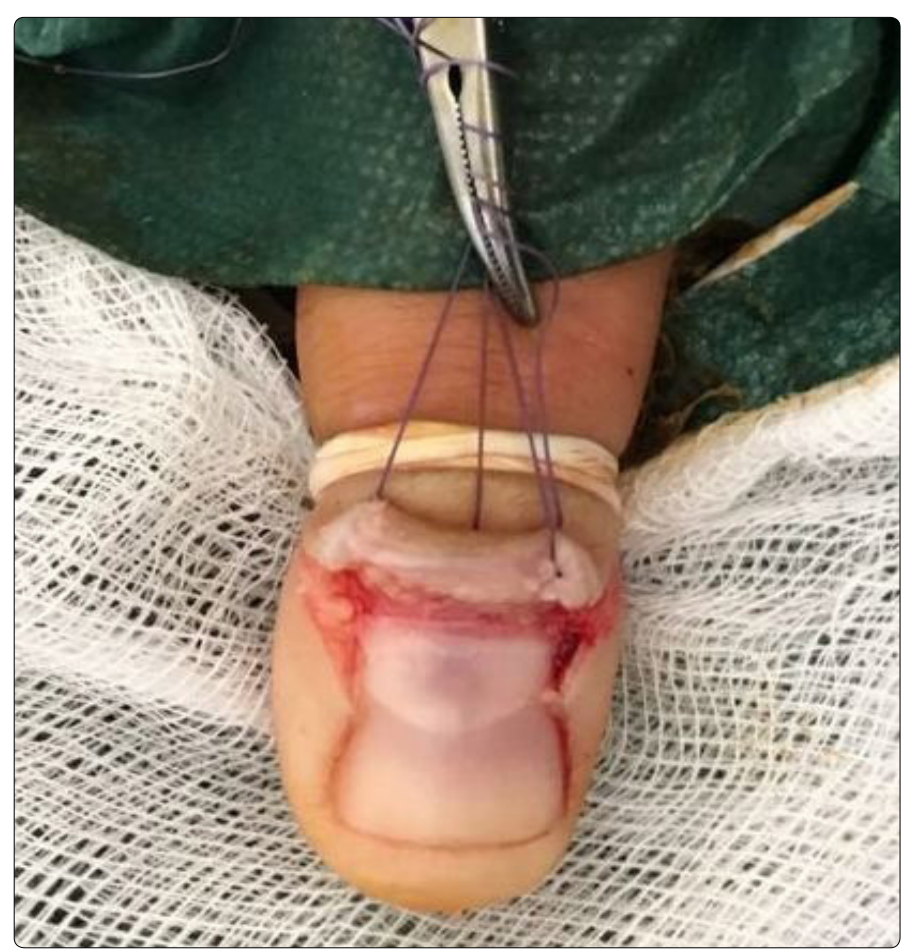

Figure 3. The lesion is exposed with the help of nail plate avulsion and retraction of proximal nail fold.

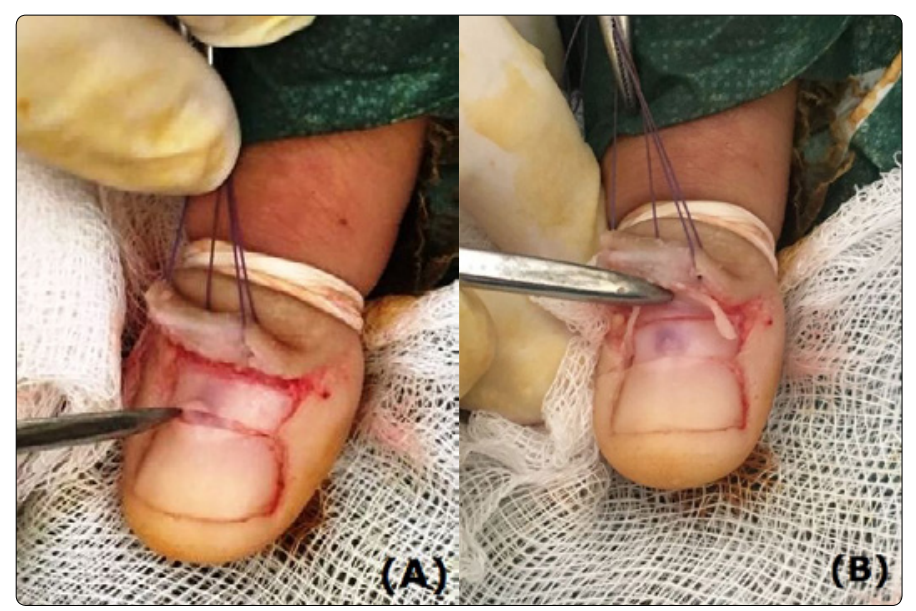

Figure 4. (A) Transverse incision in the proximal third of the nail bed. (B) Tumor exposed after partial proximal nail avulsion. 


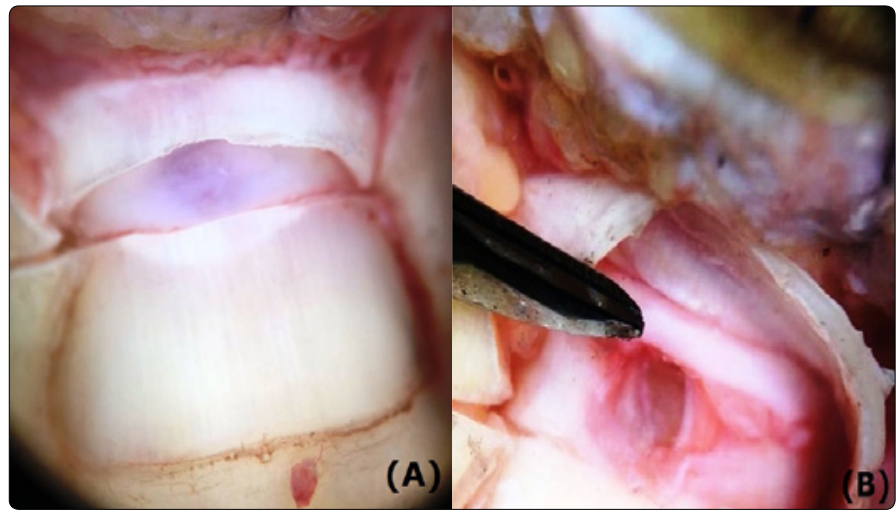

Figure 5. Intraoperative dermoscopic showing: (A) purple discoloration of the mass after nail plate incision (B): purplish-red nodule after the nail bed elevation.

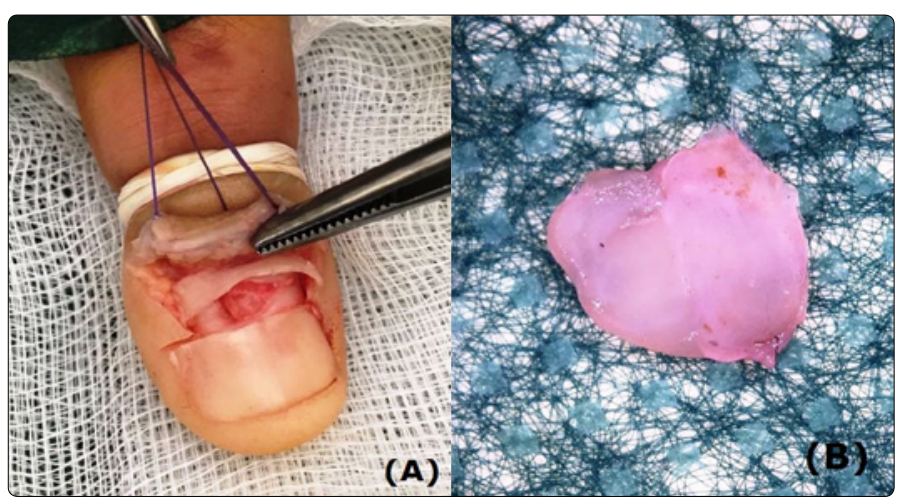

Figure 6. (A) Cavity after removal of the glomus tumor. (B) Pinkish homogeneous nodule with marked telangiectasias.

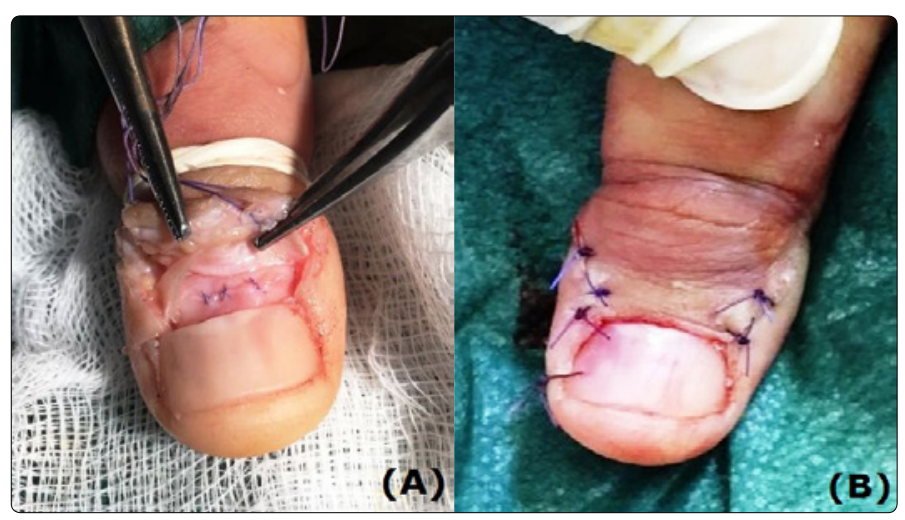

Figure 7. (A) The surgical defect is closed in a transversal fashion with 6-0 absorbable sutures. (B) The avulsed nail plate is repositioned and lateral incisions were sutured with 3-0 non-absorbable sutures.

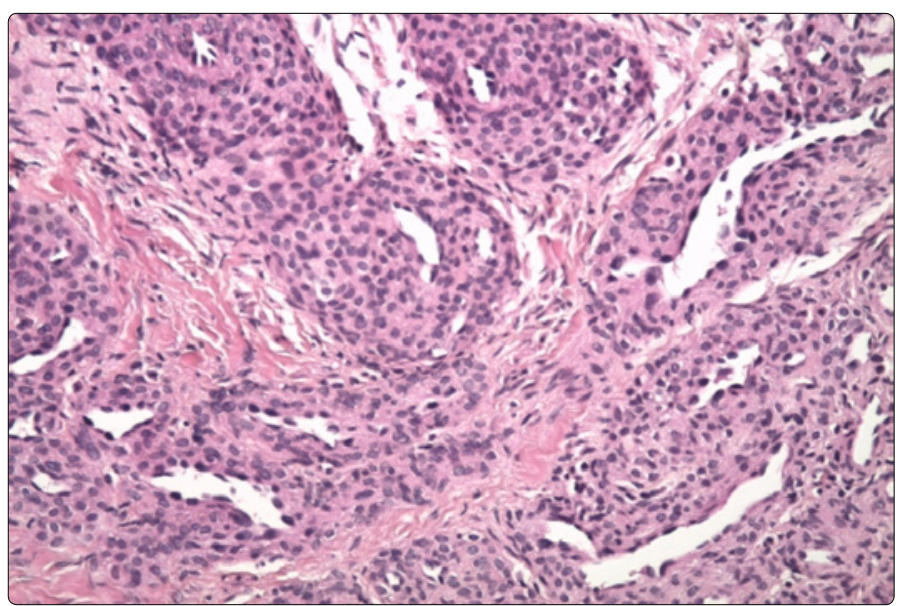

Figure 8. Nests of round uniform small cells surrounding the blood vessel walls (b) $(H E, x 200)$.

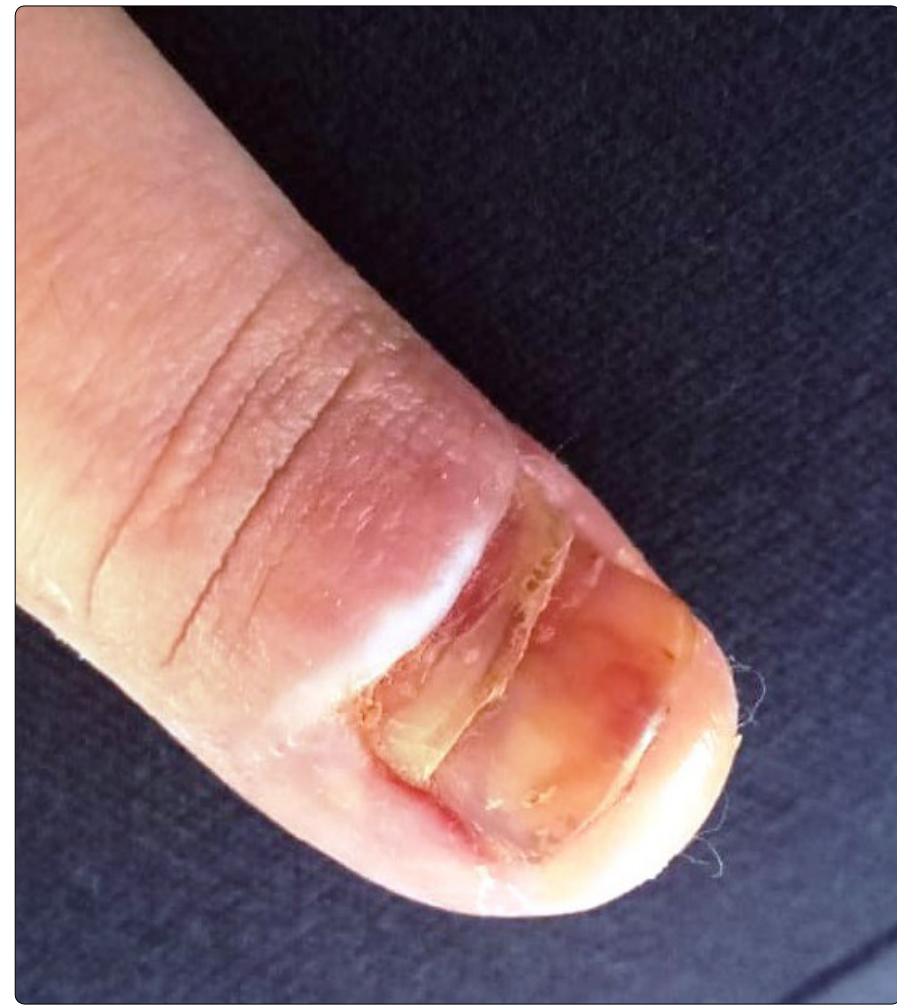

Figure 9. Control after four months: showing a healthy emergence of the nail without any deformation.

\section{Discussion}

GT is a rare, benign, vascular neoplasm arising from glomus body which is a contractile neuromyoarterial structure found in the reticular dermis [7]. It was first described by Wood in 1812 and was named by Masson in 1924 [8]. Although the exact etiology of GT is unknown, it has been proposed that a structural weakness in the glomus body could lead to reactive hypertrophy following trauma; or alternatively, they may represent hamartomas in which individual components are hypertrophied [8]. It commonly affects patients between the third and fifth decade, with a female preponderance [7].

The patients usually present with non-specific symptoms and are diagnosed late. The common symptoms include pain, sensitivity to touch, cold sensitivity, blue-reddish discoloration and nail deformity [6]. Pain is the dominating symptom and one which is most discomforting to the patient. The tumor is sensitive to temperature changes, resulting in contraction of myofilaments and rise in intracapsular pressure, which is transmitted via the unmyelinated nerve fibers leading to the perception of pain [6]. Diagnosis is made largely on a clinical basis. Two signs, named Love test and Hildreth sign, are helpful [9]. The Love's sign is positive when there is severe localized tenderness on applying pressure with a pinhead and relieved on removing the pressure. The Hildreth's sign is performed by elevating the patient's arm and then tying a tourniquet to the arm which is inflated to $250 \mathrm{mmHg}$. This result in reduction or abolition of pain in the digit with glomus tumor and the pain reappears when the tourniquet is deflated [10]. In our case, touch sensitivity and pain were the primary symptoms. 
The main differential diagnosis of subungual GT is onychopapilloma and subungual Bowen disease. Dermoscopy with gel can be very useful to obtain better visualization of the mass. The tumor appears as a deep red-purple discolored area with blurred borders, or as a band of longitudinal erythronychia that does not usually reach the distal margin [11]. The intensity of the red color contrasts with the pale pink color of the surrounding nail bed and with the white color of the lunula [12]. Nail plate dermoscopy can also show the presence of small red ramified vessels. In cases of a bigger mass, onycholysis could be present, as well as nail plate thinning and fissuring due to matrix compression [13]. A new tool for the diagnosis of glomus tumor is the "pink glow" sign visible on UV light, where a pinkish glow suggests the vascular nature of the tumor [14]. In our case, the clinical context and the presence of longitudinal erythronychia with interruption of the lunula oriented us to the diagnosis of GT.

Imaging studies such as MRI or ultrasound are not necessary in case of classical subungual GT and clinical history and examination are sufficient in diagnosing and planning the management. However, MRI and ultrasonography have a definite role when there are multiple lesions and when there is uncertainty regarding the diagnosis with lesions in unusual locations [15].

Histologically, glomus tumors are made up of three components: Glomus cells, vascular structures and smooth muscle cells. The glomus cells are positive for vimentin, and alpha-smooth muscle actin, but negative for desmin at histochemical staining [16].

Regarding therapy: Several surgical approaches have been described in literature. In the case of a matrix GT, two surgical pathways was described [17]: a transungual pathway, reported in this paper which is the technique of choice and a subperiosteal lateral approach, theoretically less deleterious, but which exposes to a higher recurrence rate and postoperative paresthesia. In this approach, the subungual region is accessed directly by a lateral incision which is curved distally along the pulp of the finger and without any nail bed dissection or disrupting the interosseous supports of the distal phalanx [18]. The transungual method with partial proximal nail avulsion as we did should be favored by making sure to place the pathway at the level of the distal matrix parallel to the free edge matrix [6]. The risk of dystrophy is exceptional. There are sometimes some residual longitudinal ridges. Any recurrence of symptoms is probably related to incomplete excision of the original lesion, rather than true recurrence of the tumor [4]. True recurrence of the tumor is rare [19].

Intraoperative localization of the tumor is a difficult task in many cases. Intraoperative dermoscopy of nail bed and matrix is an easy, rapid, and noninvasive method for proper localization of tumor. It shows the presence of ramified telangiectasias over blue background at the site of the tumor. Tumor margin can be considered corresponding to abrupt loss of telengiectesias at the periphery of mass. Proper pressure of tourniquet is important to create a bloodless field for successful intraoperative dermoscopy [20,11]. Intraoperative dermoscopy also allows, after lesion excision, the visualization of other residual macroscopic tumor foci in the nail apparatus [5]. In this case, we successfully located the tumor and predicted the approximate size of the lesion using nail plate dermoscopy. The characteristics and site of the tumor shown in the nail plate dermoscopy were identical to MRI findings. Based on these pieces of information, we selected partial nail plate retraction rather than total nail avulsion for the removal of the tumor.

\section{Conclusion}

Glomic tumors are rare but not exceptional. The diagnosis remains clinical in most cases; we suggest that pre and intraoperative dermoscopy should be performed in every case of nail bed and matrix glomus tumor. It will help to avoid incomplete excision of the tumor as well as decrease the damage to the surrounding nail matrix. MRI can be useful in doubtful cases or in case of recurrence. Surgical technique by transungual excision with Partial Proximal Nail Avulsion seems efficient and well tolerated.

\section{Conflict of Interest}

The authors confirm that there is no conflict of interest regarding this manuscript.

\section{References}

1. Baek HJ, Lee SJ, Cho KH, et al. Subungual tumors: Clinicopathologic correlation with US and MR imaging findings. Radiographics. 2010; 30(6): 1621-1636. doi: 10.1148/rg.306105514

2. Tang CY, Tipoe T, Fung B. Where is the Lesion? Glomus Tumours of the Hand. Arch Plast Surg. 2013; 40(5): 492-495. doi: 10.5999/aps.2013.40.5.492

3. Theumann NH, Goettmann S, Le Viet $D$, et al. Recurrent glomus tumors of fingertips: MR imaging evaluation. Radiology. 2002; 223(1): 143-151. doi: 10.1148/radiol.2231010977

4. Lin YC, Hsiao PF, Wu YH, Sun FJ, Scher RK. Recurrent digital glomus tumor: analysis of 75 cases. Dermatol Surg. 2010; 36(9): 1396-1400. doi: 10.1111/j.1524-4725.2010.01647.x

5. Pandey CR, Singh N, Tamang B. Subungual Glomus Tumours: Is Magnetic Resonance Imaging or Ultrasound Necessary for Diagnosis? Malays Orthop J. 2017; 11(1): 47-51. doi: 10.5704/MOJ.1703.020

6. Grover C, Khurana A, Jain R, Rathi V. Transungual Surgical Excision of Subungual Glomus Tumour. J Cutan Aesthet Surg. 2013; 6(4): 196-203. doi: 10.4103/0974-2077.123401

7. Drape JL, Idy-Peretti I, Goettmann S, et al. Subungual glomus tumours: evaluation with MR imaging. Radiology. 1995; 195(2): 507-515. doi: 10.1148/radiology.195.2.7724775

8. Samaniego E, Crespo A, Sanz A. Key diagnostic features and treatment of subungual glomus tumor. Actas Dermosifiliogr. 2009; 100(10): 875-882.

9. Hazani R, Houle MJ, Kasdan LM, Wilhelmi JB. Glomus Tumors of the Hand. Eplasty. 2008; 8: e48.

10. Bhaskaranand K, Navdag BC. Glomus tumours of the hand. J Hand Surg Br. 2002; 27(3): 229-231. doi: 10.1054/jhsb.2001.0746

11. Maehara Lde S, Ohe EM, Enokihara MY, Michalany NS, Yamada S, Hirata $\mathrm{SH}$. Diagnosis of glomus tumor by nail bed and matrix dermoscopy. An Bras Dermatol. 2010; 85: 236-238.

12. de Berker D. Erythronychia. Dermatol Ther. 2012; 25(6): 603-611. doi: 10.1111/j.1529-8019.2012.01525.x 
13. Alessandrini A, Starace M, Piraccini BM. Dermoscopy in the Evaluation of Nail Disorders. Skin Appendage Disord. 2017; 3: 70-82. dol: $10.1159 / 000458728$

14. Thatte SS, Chikhalkar SB, Khopkar US. "Pink glow": a new sign for the diagnosis of glomus tumor on ultraviolet light dermoscopy. Indian Dermatol Online J. 2015; 6: S21-S23. doi: 10.4103/2229-5178.171041

15. Mitchell A, Spinner RJ, Ribeiro A, Mafra M, Mouzinho MM, Scheithauer BW. Glomus tumor of digital nerve: case report. J Hand Surg Am. 2012; 37(6): 1180-1183. doi: 10.1016/j.jhsa.2012.02.035

16. Cogrel O, Thomas L. Chirurgie unguéale. In: Amici, Bailly JY, Beylot-Barry $M$, Egasse $D$, Thomas $L$ (eds.) Chirurgie dermatologique. Paris: Elsevier Masson SAS; 2011: 255.
17. Carroll RE, Berman AT. Glomus tumors of the hand: Review of the literature and report on twenty-eight cases. J Bone Joint Surg Am. 1972; 54(4): 691703.

18. Morey VM, Garg B, Kotwal PP. Glomus tumours of the hand: Review of literature. J Clin Orthop Trauma. 2016; 7(4): 286-291. doi: 10.1016/j. jcot.2016.04.006

19. Lee $T$, Jo G, Mun JH. The usefulness of nail plate and intraoperative dermoscopy in subungual glomus tumor. Int J Dermatol. 2018; 57(3): e26-e28. doi: 10.1111/ijd.13888

20. Rai AK. Role of intraoperative dermoscopy in excision of nail unit glomus tumor. Indian Dermatol Online J. 2016; 7(5): 448-450. doi: 10.4103/22295178.190488 